Article

\title{
Analysis of Eyring-Powell Fluid Flow Used as a Coating Material for Wire with Variable Viscosity Effect along with Thermal Radiation and Joule Heating
}

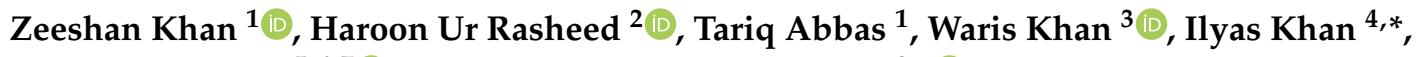 \\ Dumitru Baleanu ${ }^{5,6,7}$ (D) and Kottakkaran Sooppy Nisar $8, *$ (D) \\ 1 Sarhad University of Science and Information Technology, Peshawar, KPK 25000, Pakistan; \\ zeeshansuit@gmail.com (Z.K.); tariqabbas56@yahoo.com (T.A.) \\ 2 Department of Mathematics, Abdul Wali Khan University Mardan, KPK 23000, Pakistan; \\ haroon.csit@suit.edu.pk \\ 3 Institute of Numerical Sciences, Kohat University of Science and Technology, Kohat, KPK 26000, Pakistan; \\ wariskhan758@yahoo.com \\ 4 Department of Mathematics, College of Science Al-Zulfi, Majmaah University, Al-Majmaah 11952, \\ Saudi Arabia \\ 5 Department of Mathematics, Cankaya University, 06790 Ankara, Turkey; dumitru@cankaya.edu.tr \\ 6 Institute of Space Sciences, 077125 Magurele-Bucharest, Romania \\ 7 Department of Medical Research, China Medical University Hospital, China Medical University, \\ Taichung 40447, Taiwan \\ 8 Department of Mathematics, College of Arts and Sciences, Prince Sattam bin Abdulaziz University, \\ Wadi Aldawaser 11991, Saudi Arabia \\ * Correspondence: i.said@mu.edu.sa (I.K.); n.sooppy@psau.edu.sa (K.S.N.)
}

Received: 4 February 2020; Accepted: 1 March 2020; Published: 4 March 2020

\begin{abstract}
This article examines a wire coating technique that considers how viscoelastic Eyring-Powell fluid is studied with magnetohydrodynamic (MHD) flow, thermal transfer, and Joule heating effects. Temperature-dependent variable and flexible viscosity models are considered. The interface boundary layer equalities which describe flux and thermal convective phenomena are evaluated using a dominant numerical technique-the so-called Runge-Kutta 4th-order method. A permeable matrix which behaves like a dielectric to avoid heat dissipation is taken into account and is the distinguishing aspect of this article. The effect of thermal generation is also explained, as it controls power. The effects of various parameters, such as non-Newtonian fluid, magnetic field, permeability, and heat source/sink, on wire coating processes are investigated through graphs and explained in detail. For the sake of validity, numerical techniques are compared with a semi-numerical technique (HAM) and $\mathrm{BVPh} 2$, and an outstanding agreement is found.
\end{abstract}

Keywords: coating process; MHD flow; Eyring-Powell fluid; temperature-dependent viscosity; Joule heating

\section{Introduction}

Various fluids like air, water, and other lubricants are considered to be non-Newtonian fluids in science and engineering technologies. In many situations, the idea of Newtonian fluid behavior is not trivial, but it may be more complicated and a perturbed (non-Newtonian) model must be considered. Non-Newtonian behavior exists in different liquid materials like glue, paint, ketchup, custard, and 
blood. Because of their vast and significant uses in industry, petroleum and chemical engineering, and in other fluid mechanics, they have gained the attention of researchers [1-8].

Ellahi et al. [9] discussed the non-Newtonian micropolar liquid in blood movement over a composite stenosis. Eyring-Powell fluid is a non-Newtonian fluid which was first presented in 1944 by Eyring and Powell. Many important features of Eyring-Powell fluid are discussed by researchers [10-14]. Wire coating techniques are very important as they can help avoid injury and decrease losses which may be generated by machine oscillation. In manufacturing industries, various liquid polymers are used in wire coating processes. A polymer melt is put over a wire and then the wire is dragged over the die, covered by viscoelastic material. Three methods are used for wire coating. These methods are termed as electrostatic deposition, dipping, and coaxial processes. Compared to the other two, the dipping method provides a stronger link in the field but is very slow. It involves a payoff device, a die and extruder device, a cooling device, a preheater, a straightener, a tester, a capstan, and a take-up roll. In the process, a bare wire is set rolling over the payoff device which goes through a straightener. Next, heat is delivered to the wire by a preheater and a crosshead die which has a canonical die accumulating the liquid polymer, and the wire is coated. The temperature of the hot coated wire is reduced with a cooling device after which it goes through a capstan, then a tester, and finally, the coated wire is drawn up the take-up reel. Many other researchers [15-20] have used various non-Newtonian materials for wire coating processes. In magnetohydrodynamics (MHD), the magnetic field induces the current which has a major effect on the motion of the fluid material. In recent years, MHD has been an attractive area for researchers because of its vast usage in different industrial processes, such as those used in magnetic materials and glass manufacturing processes. Many researchers [21-28] consider MHD as a current-conducting liquid in the presence of an applied magnetic field.

Fluid flux through a permeable medium has great significance for scientists because of its broad scope across engineering technologies. Some renowned permeable media include wood, carbonate rocks, and metal foams. Many researchers [29-33] have drawn attention to permeable media. Currently, a thin permeable layer is used in different domestic and industrial applications like filters, batteries, fuel cells, and printing papers. The study of the heat convection in non-Newtonian liquids has gathered interest with time because of various uses in different industries. For example, Rehman and Nadeem [34] investigated heat convective analysis for multi-directional stagnation flow movement. Khan (along with other researchers) [35-40] has studied the effects of thermal convection and MHD fluid flow. In studying the above articles, it has been found that wire coating techniques with MHD of a viscoelastic Eyring-Powell liquid as the coating substance has not yet been discussed. This paper discusses a procedure for wire coating considering the impact of thermal generation and a permeable medium, along with temperature-dependent flexible viscosity using Vogel's and Reynolds' models [41,42].

\section{Modeling of Wire Coating}

In Figure 1, the geometry of our problem is described. Different parameters are set out, like the length of pressure die $L$, radius $R_{d}$, and the saturated temperature $\theta_{d}$ due to incompressible viscoelastic Eyring-Powell material. As the temperature of the wire reaches $\theta_{W}$, the radius equals $R_{W}$, and the velocity becomes $U_{W}$ in the permeable medium. Next, the wire is pulled over the center length of the die in a static pressure die. The out flux fluid is acted on simultaneously by uniform pressure gradient $\frac{d p}{d z}$, along the axis of the object and a magnetic field of strength $B_{0}$. The magnetic field is perpendicular to the direction of the Eyring-Powell incompressible fluid movement. To reduce or neglect the perturbation in the magnetic field, we used the Reynolds number in our problem. The wire and die have a common axis of symmetry which is also taken as a reference of the coordinated system. The appropriate expressions for the fluid velocity $\vec{q}$, the stress tensor $S$, and the field temperature for this problem can be taken as follows: 


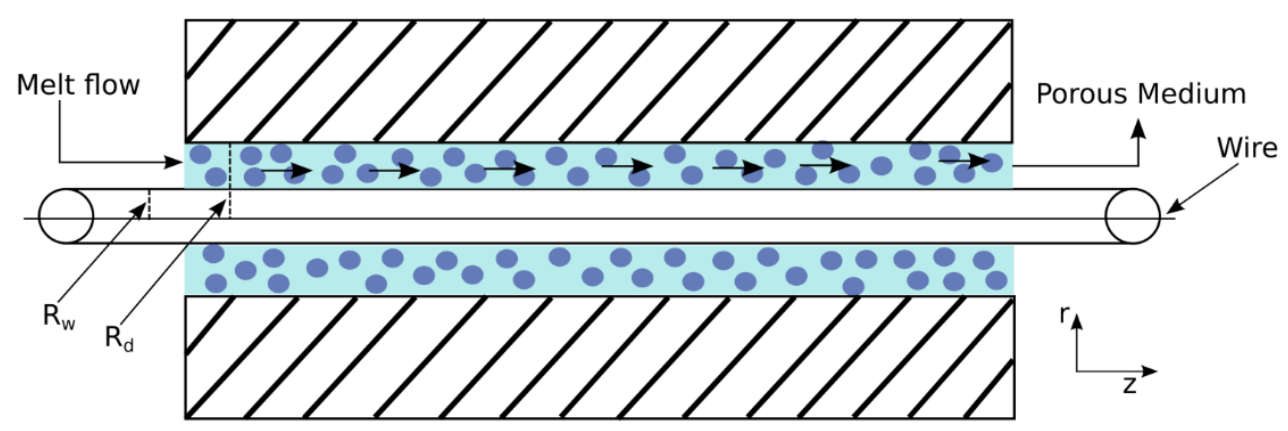

Figure 1. Geometry of the model problem.

$$
\begin{gathered}
\overrightarrow{\boldsymbol{q}}=o i+0 j+w(r) k, \\
S=S(r), \\
\theta=\theta(r) .
\end{gathered}
$$

The Cauchy stress tensor for Eyring-Powell viscoelastic fluid can be taken as

$$
S=\mu \nabla \mathrm{v}+\frac{1}{\beta} \sin ^{-1}\left(\frac{1}{C} \nabla \mathrm{v}\right)
$$

where $\mu$ denotes the viscosity, $S$ denotes the Cauchy stress tensor, $V$ is the velocity, and $C$ is the material constant. Equation (4) can be expressed as

$$
\sin ^{-1}\left(\frac{1}{C} \nabla \mathrm{v}\right)=\frac{1}{C} \nabla \mathrm{v}-\frac{1}{6}\left(\frac{1}{C} \nabla \mathrm{v}\right)^{3},\left|\frac{1}{C} \nabla v\right| \ll 1 .
$$

The boundary conditions for the proposed model take the form of:

$$
w\left(R_{w}\right)=U_{w}, \theta\left(R_{w}\right)=\theta_{w}, w\left(R_{d}\right)=0, \theta\left(R_{d}\right)=\theta_{d}
$$

Governing equations are given by:

$$
\begin{gathered}
\nabla \overrightarrow{\boldsymbol{q}}=0, \\
\rho\left(\frac{D \overrightarrow{\boldsymbol{q}}}{D t}\right)=\vec{F}-\nabla p+\vec{j} \times \vec{B}+\frac{\mu \overrightarrow{\boldsymbol{q}}}{K_{p}^{*}} \\
\rho C_{p}\left(\frac{D \overrightarrow{\mathbf{q}}}{D t}\right)=k \nabla^{2}+\varphi+Q_{0}\left(\theta-\theta_{w}\right)+j_{d}
\end{gathered}
$$

where $\overrightarrow{\boldsymbol{q}}$ is the velocity vector, $\rho$ represents density and is a temporal derivative, $\vec{j} \times \vec{B}$ indicates electromagnetic origin per unit volume appearing due to the correspondence of the magnetic arena, current $Q_{0}$ represents the rate of volumetric heat generation, and $J_{d}$ is the Joule dissipation term. The magnetic body force produced along the $z$-direction can be defined as

$$
\vec{j} \times \vec{B}=\left(0,0, \sigma \beta_{0}^{2} w\right) .
$$

Applying Equations (1)-(3), the continuity of Equation (7) is identically satisfied and we get non-vanishing components of extra stress tensor $S$ as

$$
S_{z r}=\left(\mu+\frac{1}{\beta C}\right) \frac{d w}{d r}-\frac{1}{6 \beta C^{3}}\left(\frac{d w}{d r}\right)^{3} .
$$


Putting the velocity field and Equations (10)-(11) in Equation (8), we get

$$
\begin{gathered}
\frac{\partial p}{\partial r}=0, \\
\frac{\partial p}{\partial \theta}=0, \\
\frac{\partial p}{\partial z}=\frac{1}{r} \frac{d}{d r}\left[r\left\{\left(\mu+\frac{1}{\beta C}\right) \frac{d w}{d r}-\frac{1}{6 \beta C^{3}}\left(\frac{d w}{d r}\right)^{3}\right\}\right]-\sigma \beta_{0}^{2} w-\frac{\mu w}{K_{p}^{*}} .
\end{gathered}
$$

However, Equation (14) shows the flow following the pressure gradient. When we leave the die, only drag of the wire occurred. This is why the pressure gradient is contributing nothing in the axial direction. So, Equation (14) takes the form:

$$
\frac{1}{r} \frac{d}{d r}\left[r\left\{\left(\mu+\frac{1}{\beta C}\right) \frac{d w}{d r}-\frac{1}{6 \beta C^{3}}\left(\frac{d w}{d r}\right)^{3}\right\}\right]-\sigma \beta_{0}^{2} w-\frac{\mu w}{K_{p}^{*}}=0,
$$

the energy Equation (9) becomes

$$
\begin{aligned}
& K\left(\frac{d^{2} \theta}{d r^{2}}+\frac{1}{r} \frac{d \theta}{d r}\right)+\left(\left(\mu+\frac{1}{\beta C}\right) \frac{d w}{d r}-\frac{1}{6 \beta C^{3}}\left(\frac{d w}{d r}\right)^{3}\right) \cdot \frac{d w}{d r} \\
& +Q_{0}\left(\theta-\theta_{w}\right)+\sigma \beta_{0}^{2} w=0 .
\end{aligned}
$$

and the dimensionless parameters are defined as

$$
\begin{aligned}
& r^{*}=\frac{r}{R_{w}}, w^{*}=\frac{w}{U_{w}}, M^{2}=\frac{\sigma \beta_{0}^{2} R_{w}^{2}}{\mu}, K_{p}=\frac{R_{w}^{2}}{K_{p}^{*}}, w=\frac{v_{0}}{U_{w}}, N=\frac{1}{\mu \beta C}, \theta^{*}=\frac{\left(\theta-\theta_{w}\right)}{\left(\theta_{d}-\theta_{w}\right)}, \\
& Q=\frac{Q_{0} R_{w}^{2}}{K}, B r=\frac{\mu U_{w}^{2}}{K\left(\theta_{d}-\theta_{w}\right)}, R_{w}=\frac{\beta v_{0}}{\mu}, \varepsilon=\frac{\mu}{6 w^{2}(\beta C)^{3}} .
\end{aligned}
$$

Using these new variables in Equations (15) and (16) with Equation (6), and after removing asterisks, we get the following form:

$$
\begin{gathered}
(1+N)\left(r \frac{d^{2} w}{d r^{2}}+\frac{d w}{d r}\right)-\varepsilon\left(\left(\frac{d w}{d r}\right)^{3}+3 r\left(\frac{d w}{d r}\right)^{2} \frac{d^{2} w}{d r^{2}}\right)-M^{2} w r-K_{p} w r=0, \\
w(1)=1 \text { and } w(\delta)=0 \\
\frac{d^{2} \theta}{d r^{2}}+\frac{1}{r} \frac{d \theta}{d r}+B_{r}(1+N)\left(\frac{d w}{d r}\right)^{2}+\varepsilon B_{r}\left(\frac{d w}{d r}\right)^{4}+Q \theta+B_{r} M^{2} w^{2}=0, \\
\theta(1)=0 \text { and } \theta(\delta)=1 .
\end{gathered}
$$

\section{Temperature-Dependent Viscosity}

Two basic models are used for the temperature-dependent viscosity: Reynolds' model and Vogel's model. The details of both are given below.

\subsection{Reynolds' Model}

Here, we have used Reynolds' model to explain temperature-dependent viscosity. The temperature-dependent viscosity for the Reynolds model can be expressed by the following relation:

$$
\mu=1-\beta_{0} m \theta
$$


This will be applied for the variation of temperature-dependent viscosity, while $m$ is used for viscosity parameter. The following equation uses nondimensional parameters:

$$
\begin{aligned}
& r^{*}=\frac{r}{R_{w}}, w^{*}=\frac{w}{U_{w}}, M^{2}=\frac{\sigma \beta_{0}^{2} R_{w}^{2}}{\mu_{0}}, K_{p}=\frac{R_{w w}^{2}}{K_{p}^{*}}, w=\frac{v_{0}}{U_{w}}, N=\frac{1}{\mu_{0} \beta C}, \mu^{*}=\frac{\mu}{\mu_{0}}, \\
& \theta^{*}=\frac{\left(\theta-\theta_{w}\right)}{\left(\theta_{d}-\theta_{w}\right)}, Q=\frac{Q_{0} R_{w}^{2}}{K}, B r=\frac{\mu_{0} U_{w}^{2}}{K\left(\theta_{d}-\theta_{w v}\right)}, R_{w}=\frac{\beta v_{0}}{\mu_{0}}, \varepsilon=\frac{\mu_{0}}{6 w^{2}(\beta C)^{3}} .
\end{aligned}
$$

After removing asterisks, we obtain a nondimensional form of a momentum and energy equation along boundary conditions:

$$
\begin{gathered}
\frac{d^{2} w}{d r^{2}}\left(r\left(1-\beta_{0} m \theta\right)+r N-3 r \varepsilon\left(\frac{d w}{d r}\right)^{2}\right)+\frac{d w}{d r}\left(1-\beta_{0} m \theta+N-\beta_{0} m r \frac{d \theta}{d r}\right) \\
-\varepsilon\left(\frac{d w}{d r}\right)^{3}-K_{p} w r-M^{2} w r=0, \\
w(1)=1 \text { and } w(\delta)=0, \\
\frac{d^{2} \theta}{d r^{2}}+\frac{1}{r} \frac{d \theta}{d r}+\left(1-\beta_{0} m \theta\right) B_{r}\left(\frac{d w}{d r}\right)^{2}+B_{r}\left(\frac{d w}{d r}\right)^{2}(N+\varepsilon)+Q \theta+B_{r} M^{2} w^{2}=0, \\
\theta(1)=0 \text { and } \theta(\delta)=1 .
\end{gathered}
$$

3.2. Vogel's Model

In this case, we take temperature-dependent viscosity as:

$$
\mu=\mu_{0} \exp \left(\frac{D}{B^{\prime}+\theta}-\theta_{w}\right) .
$$

Applying expansions, we get:

$$
\mu=\Omega\left(1-\frac{D \theta}{\left(B^{\prime}\right)^{2}}\right),
$$

where $D, B$ are parameters of viscosity and

$$
\Omega=\mu_{0} \exp \left(\frac{D}{\left(B^{\prime}\right)^{2}}-\theta_{w}\right) .
$$

We obtain nondimensional equations of momentum and energy along boundary conditions after removing asterisks:

$$
\begin{gathered}
\frac{d^{2} w}{d r^{2}}\left(r \Omega\left(1-\frac{D \theta}{\left(B^{\prime}\right)^{2}}\right)+r N-3 r \varepsilon\left(\frac{d w}{d r}\right)^{2}\right)+\frac{d w}{d r}\left(\begin{array}{c}
\Omega\left(1-\frac{D \theta}{\left(B^{\prime}\right)^{2}}\right)+N \\
-\Omega \frac{D}{\left(B^{\prime}\right)^{2}} r \frac{d \theta}{d r}
\end{array}\right) \\
-\varepsilon\left(\frac{d w}{d r}\right)^{3}-K_{p} w r-M^{2} w r=0, \\
w(1)=1 \text { and } w(\delta)=0, \\
\frac{d^{2} \theta}{d r^{2}}+\frac{1}{r} \frac{d \theta}{d r}+\Omega\left(1-\frac{D \theta}{\left(B^{\prime}\right)^{2}}\right) B_{r}\left(\frac{d w}{d r}\right)^{2}+B_{r}\left(\frac{d w}{d r}\right)^{2}(N+\varepsilon)+Q \theta+B_{r} M^{2} w^{2}=0, \\
\theta(1)=0 \text { and } \theta(\delta)=1 .
\end{gathered}
$$

\section{Solution Procedure via Runge-Kutta 4th-Order Method}

Equations (24)-(27) in the case of Reynolds' model and Equations (30)-(33) for Vogel's model are solved by using the Runge-Kutta 4th-order method along with shooting techniques. First of 
all, the above governing higher-order differential equations are converted into first-order ordinary differential equations.

Equations (24) and (26) can be written as follows:

$$
\begin{gathered}
\frac{d^{2} w}{d r^{2}}=\frac{\varepsilon\left(\frac{d w}{d r}\right)^{3}-(1+N) \frac{d w}{d r}+M^{2} w r+K_{p} w r}{(1+N) r+3 r \varepsilon\left(\frac{d w}{d r}\right)^{2}} \\
\frac{d^{2} \theta}{d r^{2}}=-\left[\frac{1}{r} \frac{d \theta}{d r}+B_{r}(1+N)\left(\frac{d w}{d r}\right)^{2}+\varepsilon B_{r}\left(\frac{d w}{d r}\right)^{4}+Q \theta+B_{r} M^{2} w^{2}\right] .
\end{gathered}
$$

Defining new variables to convert higher-order ordinary differential equations into first-order ones are as follows:

$$
\begin{gathered}
w=z_{1}, w^{\prime}=z_{2}, w^{\prime \prime}=z_{2}^{\prime} \text { and } \theta=z_{3}, \theta^{\prime}=z_{4}, \theta^{\prime \prime}=z_{4^{\prime}}^{\prime} \\
z_{2}^{\prime}=\frac{\varepsilon\left(z_{2}\right)^{3}-(1+N) z_{2}+M^{2} z_{1} r+K_{p} z_{1} r}{(1+N) r+3 r \varepsilon\left(z_{2}\right)^{2}}, \\
z_{4}^{\prime}=-\left[\frac{1}{r} z_{4}+B_{r}(1+N)\left(z_{2}\right)^{2}+\varepsilon B_{r}\left(z_{2}\right)^{4}+Q z_{3}+B_{r} M^{2} z_{1}^{2}\right] .
\end{gathered}
$$

The boundary conditions given in (25) and (27) are changed into initial conditions as

$$
\begin{aligned}
& z_{1}(1)=1 \text { and } z_{1}(\delta)=0, \\
& z_{3}(1)=0 \text { and } z_{3}(\delta)=1 .
\end{aligned}
$$

\subsection{Reynolds Model}

Equations (30) and (32) may be written after using (36) as

$$
\begin{gathered}
z_{2}^{\prime}=\frac{\varepsilon\left(z_{2}\right)^{3}+K_{p} z_{1} r+M^{2} z_{1} r-z_{2}\left(1-\beta_{0} m z_{3}+N-\beta_{0} m r z_{4}\right)}{\left(r\left(1-\beta_{0} m z_{3}\right)+r N-3 r \varepsilon\left(z_{2}\right)^{2}\right)}, \\
z_{4}^{\prime}=-\frac{1}{r}\left[z_{4}+\left(1-\beta_{0} m z_{3}\right) B_{r}\left(z_{2}\right)^{2}+B_{r}\left(z_{2}\right)^{2}(N+\varepsilon)+Q z_{3}+B_{r} M^{2} z_{1}^{2}\right] .
\end{gathered}
$$

The boundary conditions given in (31) and (33) are changed into initial conditions as

$$
\begin{aligned}
& z_{1}(1)=1 \text { and } z_{1}(\delta)=0, \\
& z_{3}(1)=0 \text { and } z_{3}(\delta)=1 .
\end{aligned}
$$

4.2. Vogel's Model

Using (36) in (30) and (32), we get the desired first-order differential equation as

$$
\begin{gathered}
z_{2}^{\prime}=\frac{\varepsilon\left(z_{2}\right)^{3}+K_{p} z_{1} r+M^{2} z_{1} r-z_{2}\left(\Omega\left(1-\frac{D z_{3}}{\left(B^{\prime}\right)^{2}}\right)+N-\Omega \frac{D}{\left(B^{\prime}\right)^{2}} r z_{4}\right)}{r \Omega\left(1-\frac{D z_{3}}{\left(B^{\prime}\right)^{2}}\right)+r N-3 r \varepsilon\left(z_{2}\right)^{2}}, \\
z_{4}^{\prime}=-\left[\frac{1}{r} z_{4}+\Omega\left(1-\frac{D z_{3}}{\left(B^{\prime}\right)^{2}}\right) B_{r}\left(z_{2}\right)^{2}+B_{r}\left(z_{2}\right)^{2}(N+\varepsilon)+Q z_{3}+B_{r} M^{2} z_{1}^{2}\right] .
\end{gathered}
$$

This is given along with the transformed boundary conditions: 


$$
\begin{aligned}
& z(1)=1 \text { and } z_{1}(\delta)=0 \\
& z_{3}(1)=0 \text { and } z_{3}(\delta)=1
\end{aligned}
$$

\section{Validation of the Method}

To validate our numerical solution, we can make the following comparison which proves the thoroughness of the proposed method. Our comparison is illustrated in Tables 1 and 2. An excellent agreement is noted between Runge-Kutta 4th-order method, the semi-numerical method HAM, and BVPh2.

Table 1. Comparison of numerical, HAM, and BVPh2 methods for the velocity profile.

\begin{tabular}{cccc}
\hline $\boldsymbol{w}(\boldsymbol{r})$ & Numerical Solution & HAM Solution & BVPh2 \\
\hline 1.0 & 1.0000000000 & 1.0000000000 & 1.0000000000 \\
\hline 1.2 & 0.6369559249 & 0.6369559343 & 0.6369559239 \\
\hline 1.4 & 0.4274349292 & 0.4274349325 & 0.4274349352 \\
\hline 1.6 & 0.3071882095 & 0.3071882321 & 0.3071882632 \\
\hline 1.8 & 0.1968612792 & 0.1968612624 & 0.1968612547 \\
\hline 2.0 & 0.00000000000 & 0.00000000000 & 0.00000000000 \\
\hline
\end{tabular}

Table 2. Comparison of numerical, HAM, and BVPh2 methods for the temperature profile.

\begin{tabular}{cccc}
\hline $\boldsymbol{\theta}(\boldsymbol{r})$ & Numerical Solution & HAM Solution & BVPh2 \\
\hline 1.0 & 0.00000000000 & 0.00000000000 & 0.00000000000 \\
\hline 1.2 & 0.9500451152 & 0.9500451271 & 0.9500451321 \\
\hline 1.4 & 1.9209191828 & 1.9209191651 & 1.9209191658 \\
\hline 1.6 & 2.7196458893 & 2.7196458689 & 2.7196458567 \\
\hline 1.8 & 2.7456308985 & 2.7456308634 & 2.7456308695 \\
\hline 2.0 & 1.00000000000 & 1.00000000000 & 1.00000000000 \\
\hline
\end{tabular}

\section{Results and Discussion}

In this paper, for coating a wire, we considered the Eyring-Powell fluid. The method of coating a wire takes place in a die with a constant magnetic field and thermal generation effects in permeable media. Different measurable emerging parameters which called the non-Newtonian parameter $\beta_{0}$, like the viscosity parameter $m$, the thermal generation parameters $Q$ and $\Omega$, for Reynolds' and Vogel's models, permeable medium parameter $K_{p}$, Brinkman number $B_{r}$, and other parameters $D$ and $M$ respectively on velocity and temperature profiles which are explained through graphs. The geometry of the problem is explained in Figure 1. Figure 2 displays the Brinkmen number $B_{r}$ over velocity distribution for Reynolds' model and it is observed that the velocity profile shows an increasing behavior with increasing $B_{r}$. In Figure 3, the results of the permeability parameter $K_{p}$ on the velocity distribution for Reynolds' model were investigated when $M=0.6, Q=0.1, \beta_{0}=0.1, m=0.3$ and $B_{r}=0.1$. From this it is noticed that the velocity profile decreases with increasing values of $K_{p}$. By raising the value of $N$, the velocity curve shows a decreasing behavior as is predicted in Figure 4 for Reynolds' model. In Figure 5, it is observed that by increasing the Brinkman number $B_{r}$ for Vogel's model, the velocity profile increases when some parameters are fixed. Figure 6 represents the velocity profile for different values of $D$. Note that the velocity profiles increase with an increasing value of $D$. In Figure 7, it is shown that taking $D=0.2, B_{r}=0.2$ and $M=0.11$, the velocity profile rises as $Q$ rises in Vogel's model. 
Figure 8 describes the inequality in temperature profile as a result of $\varepsilon$ for uniform viscosity while keeping other parameters fixed. The velocity profile decreases as the value of $\varepsilon$ increases. Figure 9 illustrates the output of $B_{r}$ on temperature distribution for uniform viscosity. Increasing the value of $B_{r}$ decreases the velocity profile. In Figure 10, the impact of $Q$ on the temperature profile is observed while the viscosity and other parameters are kept constant. Increasing the value of $Q$ causes an increase in the velocity profile. In Figure 11, it is observed that the temperature curve increases by increasing $\varepsilon$ for Reynolds' model. Figure 12 shows that for an increment in $M$ for Reynolds' model, the temperature profile increases. A decreasing behavior in the velocity curve is observed in Figure 13 at the same rate as $Q$ increases. Figure 14 shows that the temperature profile increases as the values of $M$ increase in the case of Vogel's model. In Figure 15, a decreasing behavior in temperature profile is observed when $\Omega$ is increased in Vogel's model, keeping $N=0.2, B^{\prime}=1.3, K_{p} 0.1$ and $D=0.3$.

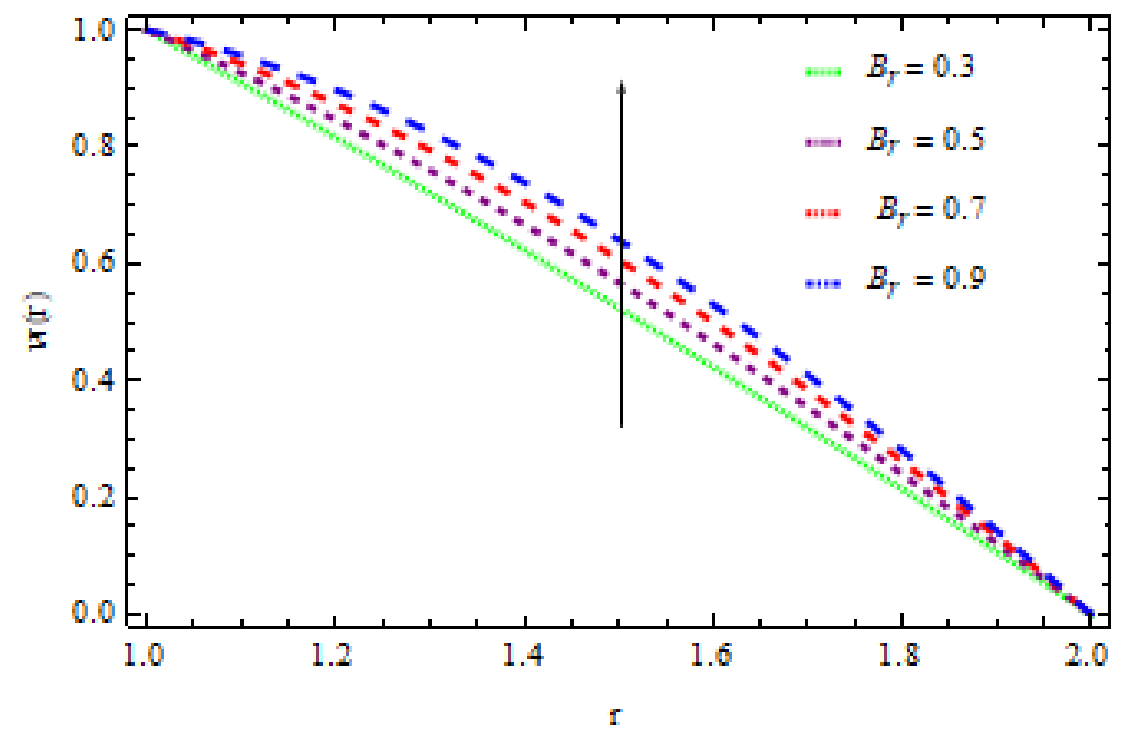

Figure 2. The influence of $B_{r}$ on velocity distribution in the case of Reynolds' model (RM) when $Q=0.2, K_{p}=0.1, M=0.1, \varepsilon=0.6, N=0.1, m=0.1, \beta_{0}=0.1$.

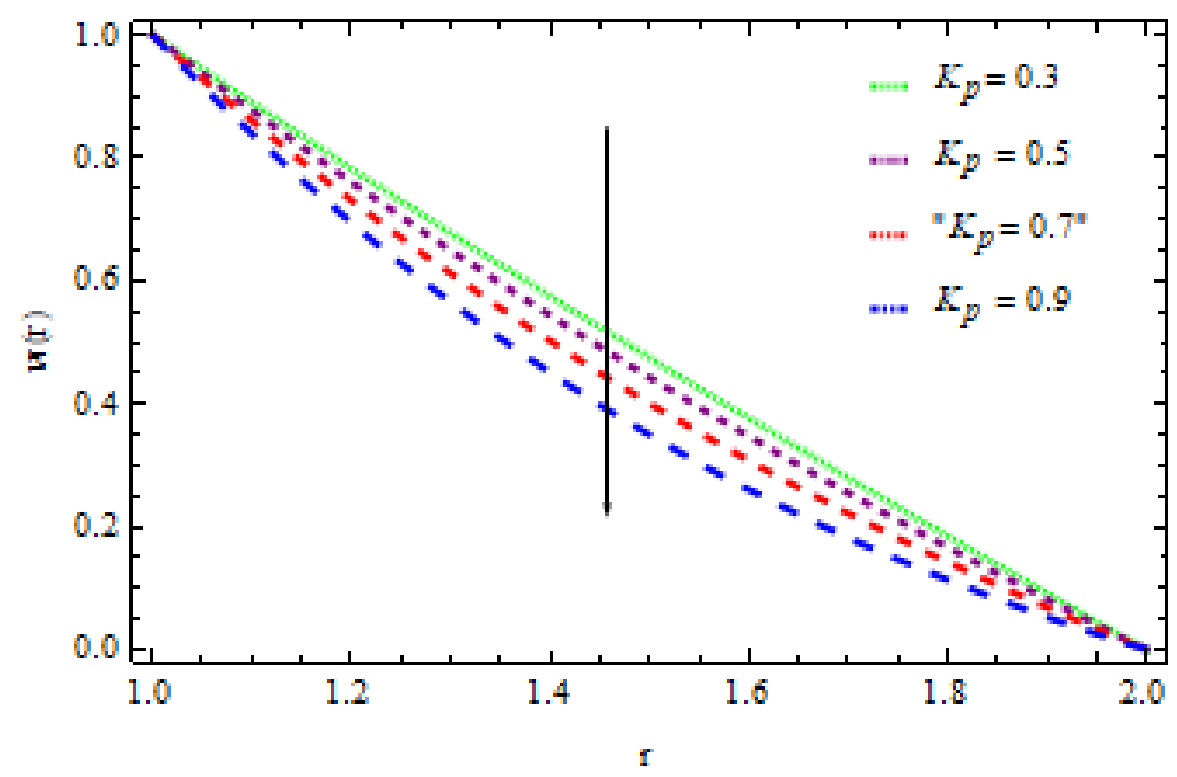

Figure 3. The influence of $K_{p}$ on velocity distribution in Reynolds' model (RM) when $Q=0.2, M=$ $0.5, \varepsilon=0.6, N=0.1, m=0.4, \beta_{0}=0.1, B_{r}=0.1$. 


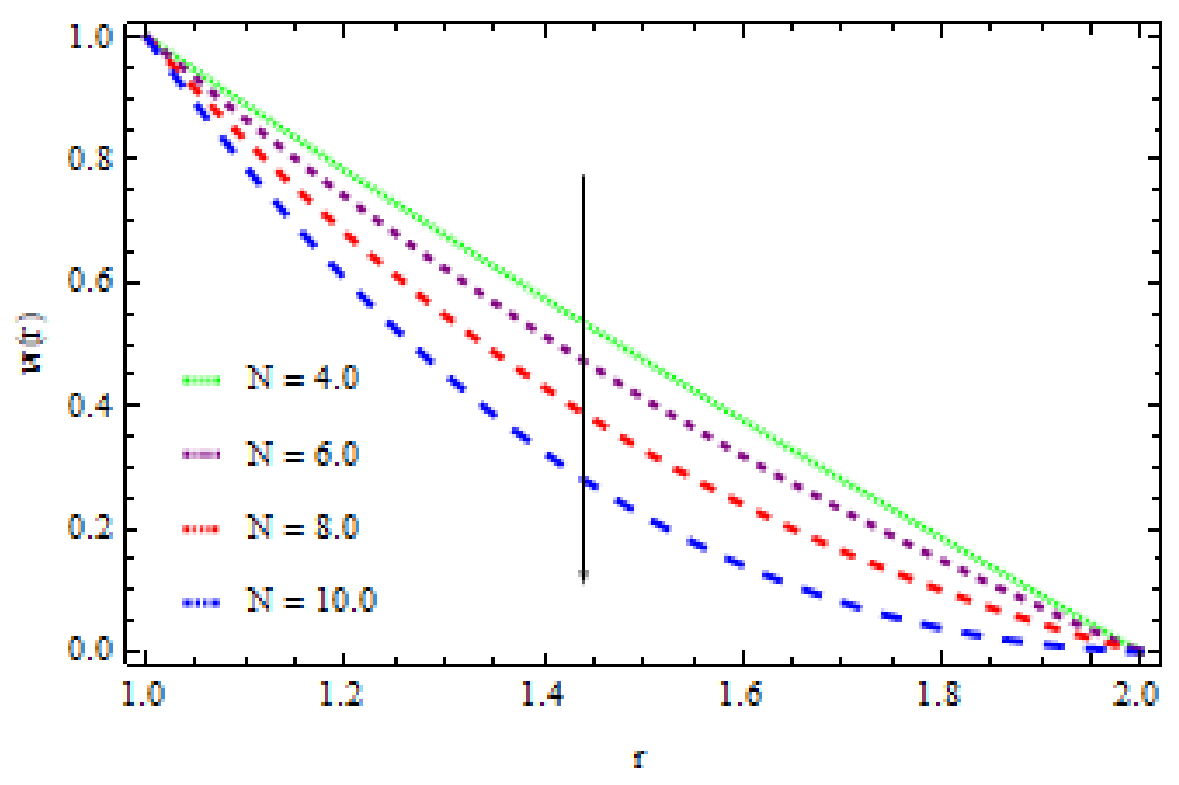

Figure 4. The influence of $N$ on velocity distribution in the case of Reynold's model (RM) when $Q=0.3, B_{r}=0.1, M=0.5, \varepsilon=0.8, m=0.13, \beta_{0}=1.2, K_{p}=0.2$.

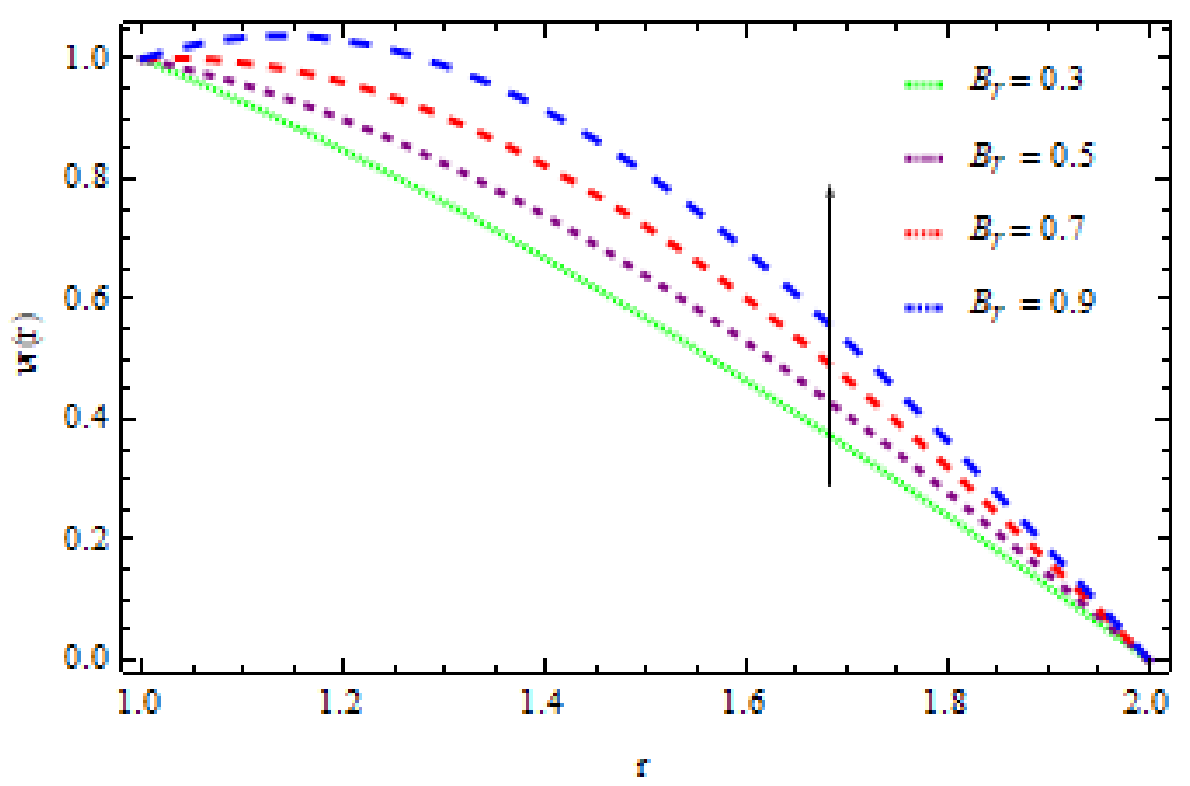

Figure 5. The influence of $B_{r}$ on velocity distribution in the case of Vogel's model (VM) when $Q=0.3, B^{\prime}=1.0, M=0.12, N=0.3, D=0.2, \Omega=1.0, K_{p}=0.2$. 


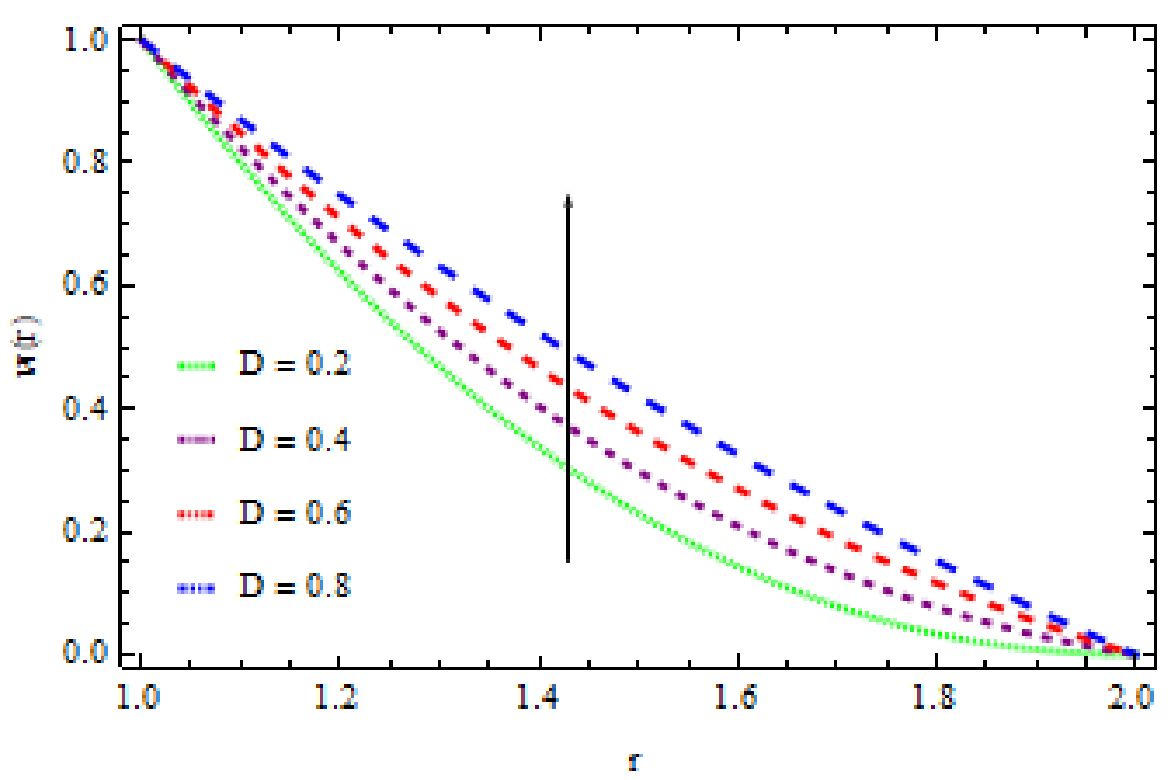

Figure 6. The influence of $D$ on velocity distribution in the case of Vogel's model (VM) when $Q=0.3, B^{\prime}=0.1, M=0.12, N=0.5, B_{r}=0.6, \Omega=1.2, K_{p}=0.2$.

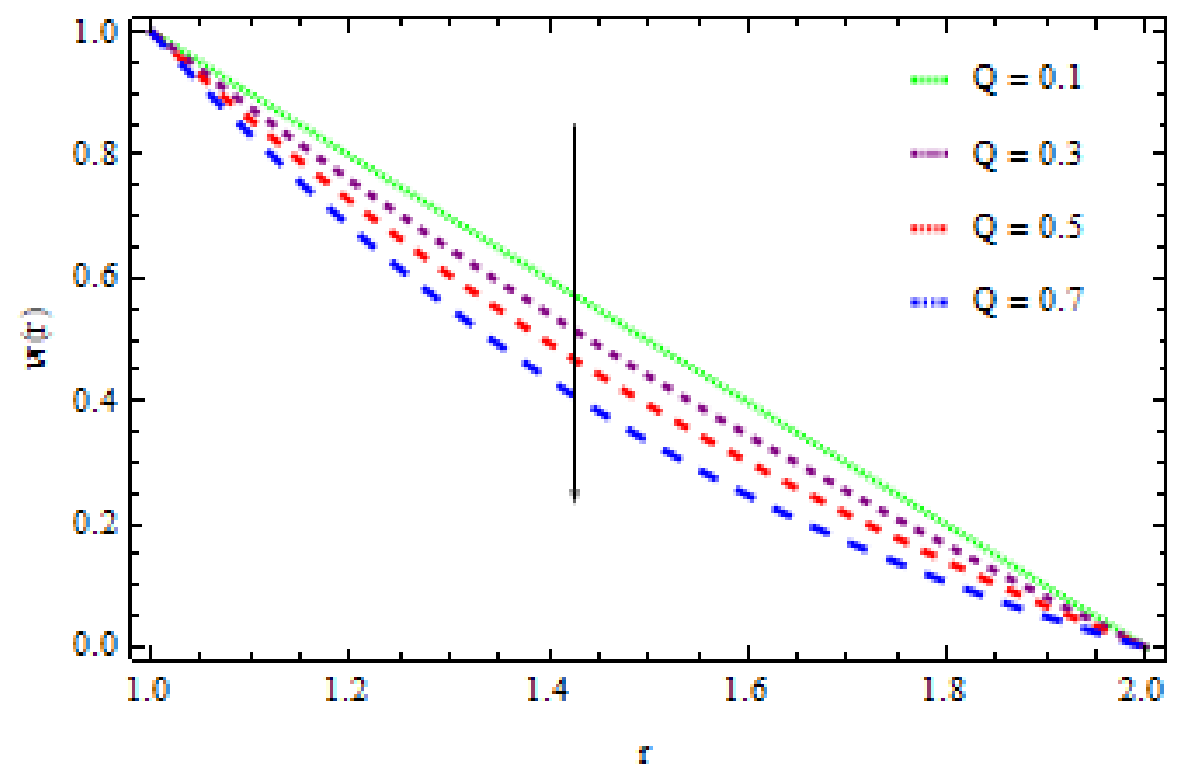

Figure 7. The influence of $Q$ on velocity distribution for Vogel's model (VM) when $B_{r}=0.2, B^{\prime}=$ $1.0, M=0.12, N=0.3, D=0.2, \Omega=1.0, K_{p}=0.2$. 


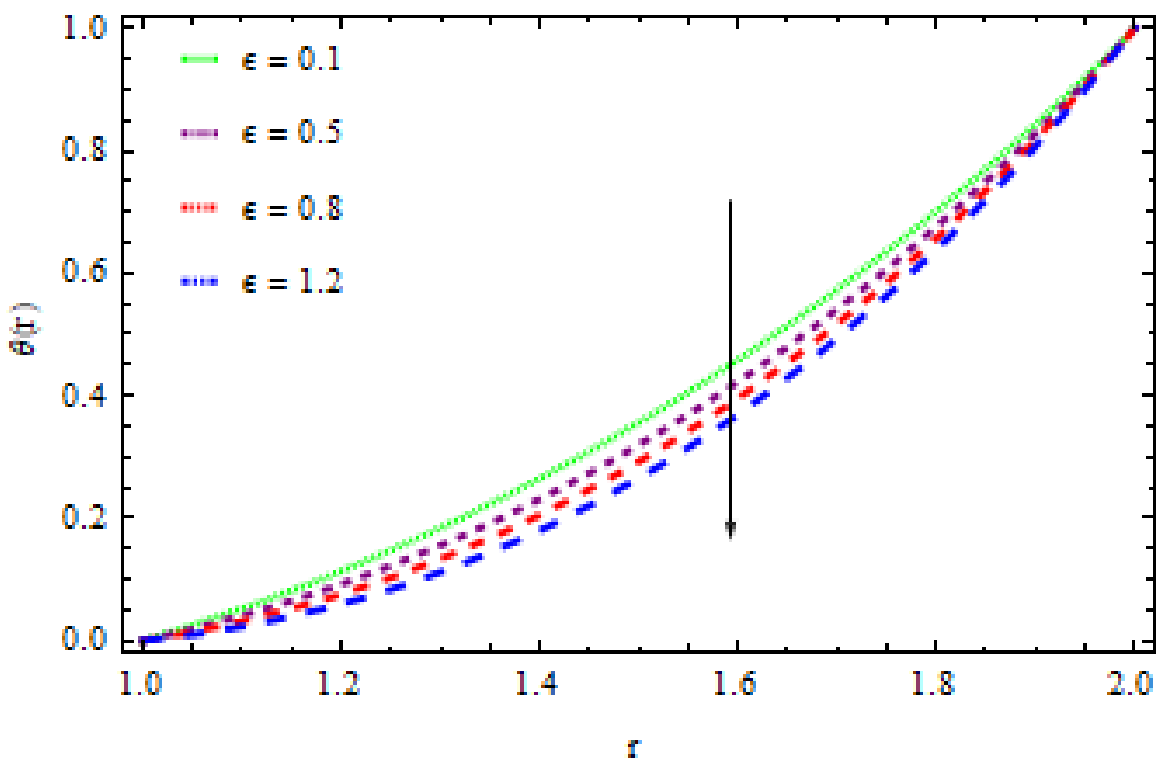

Figure 8. The influence of $\varepsilon$ on temperature when $Q=0.6, B_{r}=1.4, M=0.6, N=0.01, K_{p}=0.6$.

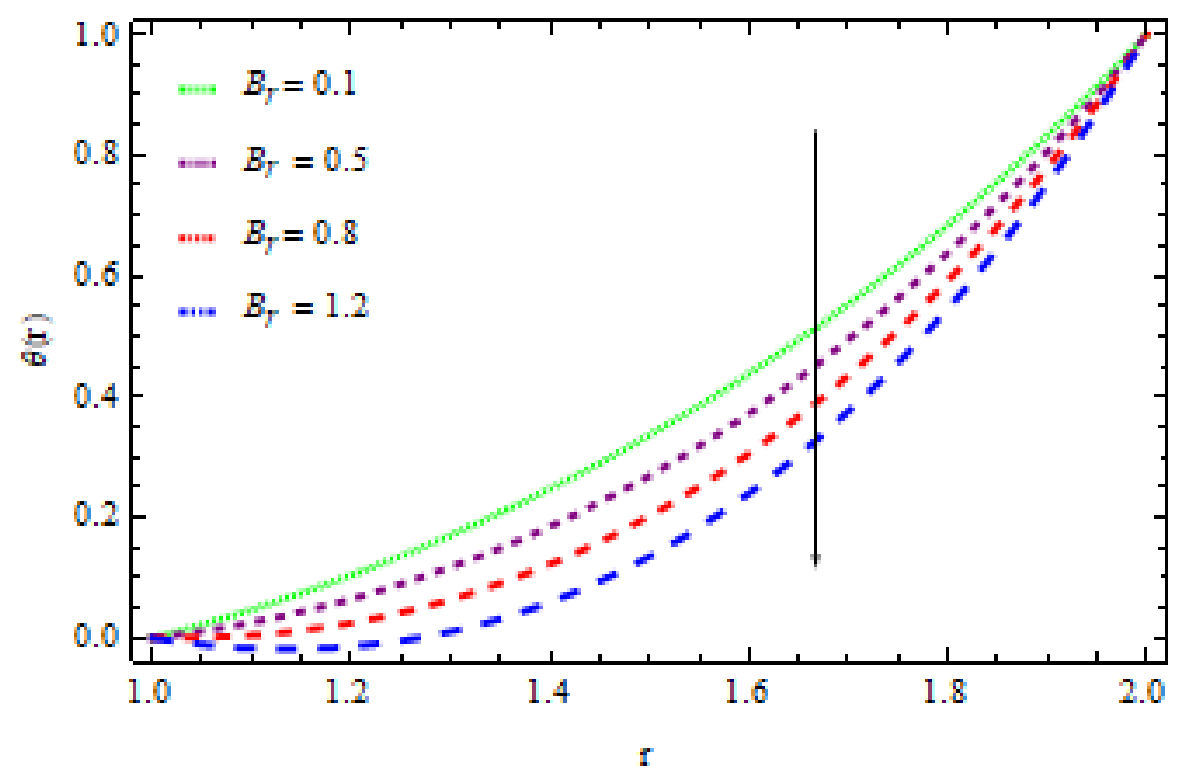

Figure 9. The influence of $B_{r}$ on temperature when $Q=0.6, \varepsilon=1.5, M=0.6, N=0.2, K_{p}=0.1$. 


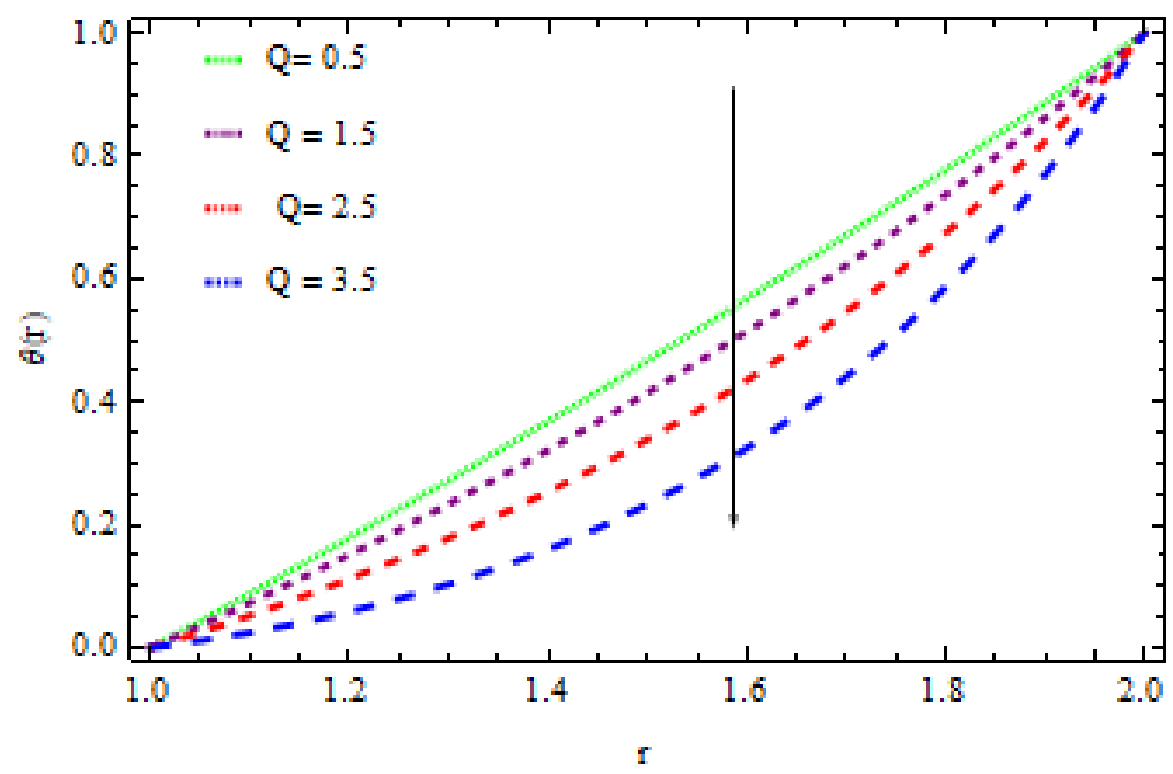

Figure 10. The influence of $Q$ on temperature when $B_{r}=0.1, M=0.6, N=0.2, K_{p}=0.5, \varepsilon=1.5$.

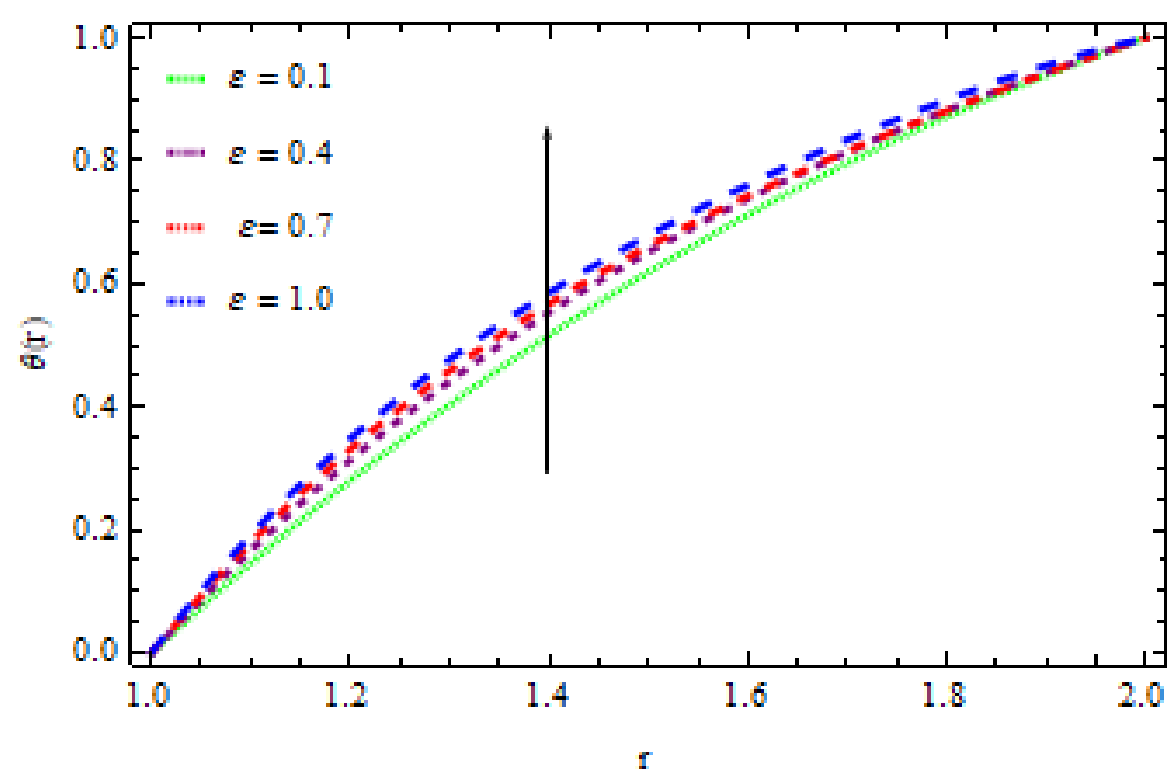

Figure 11. The influence of $\varepsilon$ on temperature distribution for Reynolds' model (RM) when $\beta_{0}=$ $1.2, m=0.1, Q=0.1, B_{r}=0.5, M=0.5, N=0.3, K_{p}=0.4$. 


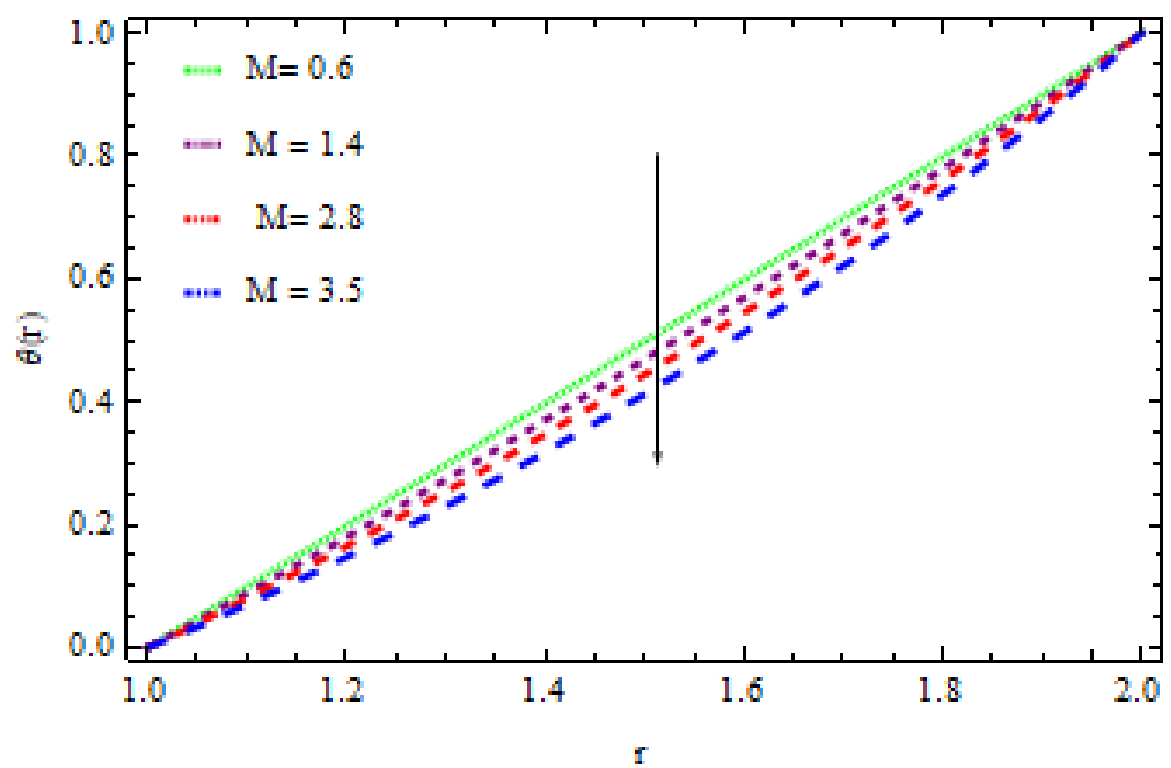

Figure 12. The influence of $M$ on temperature distribution for Reynolds' model (RM) when $\beta_{0}=$ $1, m=0.1, Q=0.1, B_{r}=0.1, \varepsilon=0.5, N=0.1, K_{p}=0.1$.

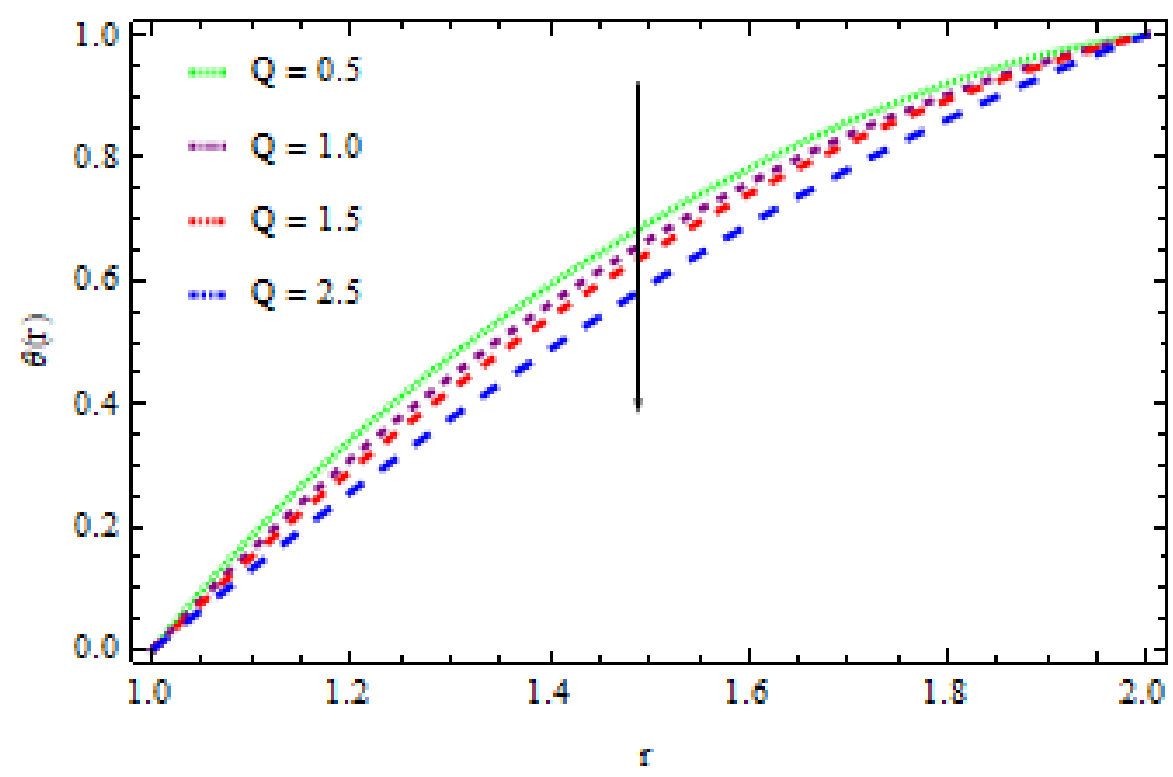

Figure 13. The influence of $Q$ on temperature distribution in the case of Reynold's model (RM) when $\beta_{0}=2.1, m=0.2, \varepsilon=0.2, B_{r}=0.5, M=0.6, N=0.2, K_{p}=0.1$. 


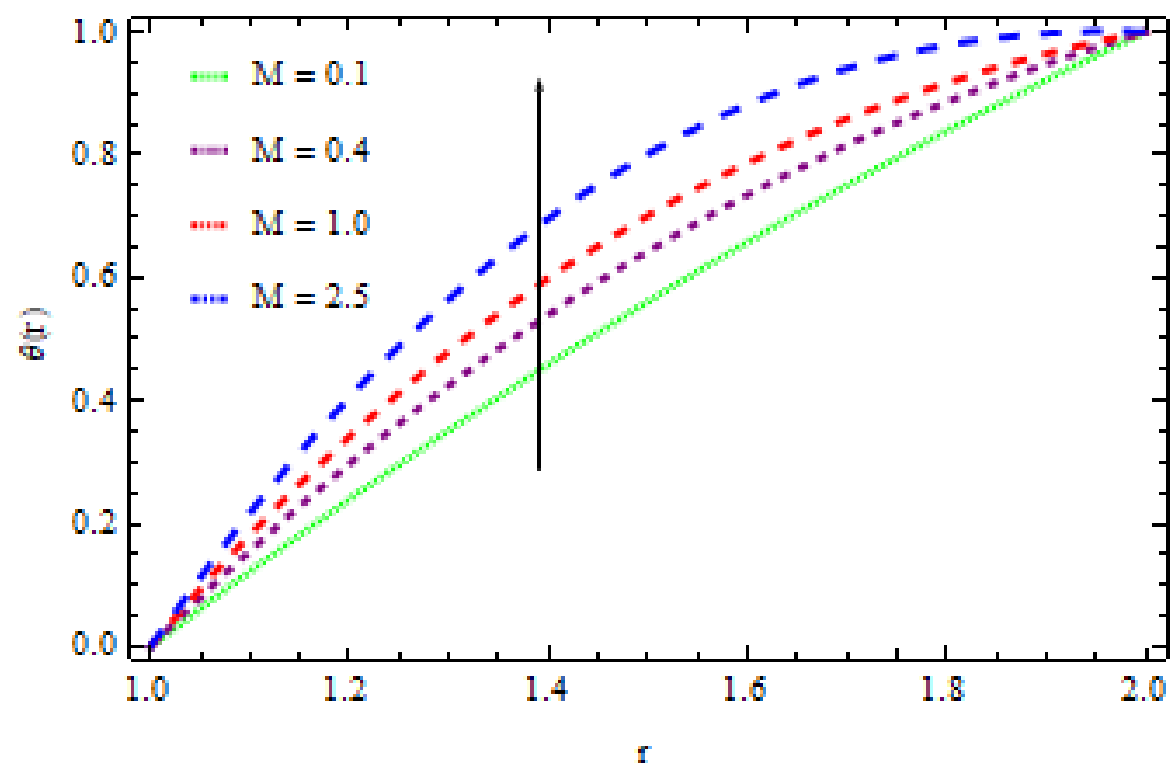

Figure 14. The influence of $M$ on temperature distribution in the case of Vogel's model (VM) when $\Omega=1.2, D=0.2, Q=0.6, B_{r}=0.5, B^{\prime}=1.1, N=0.1, K_{p}=0.1$.

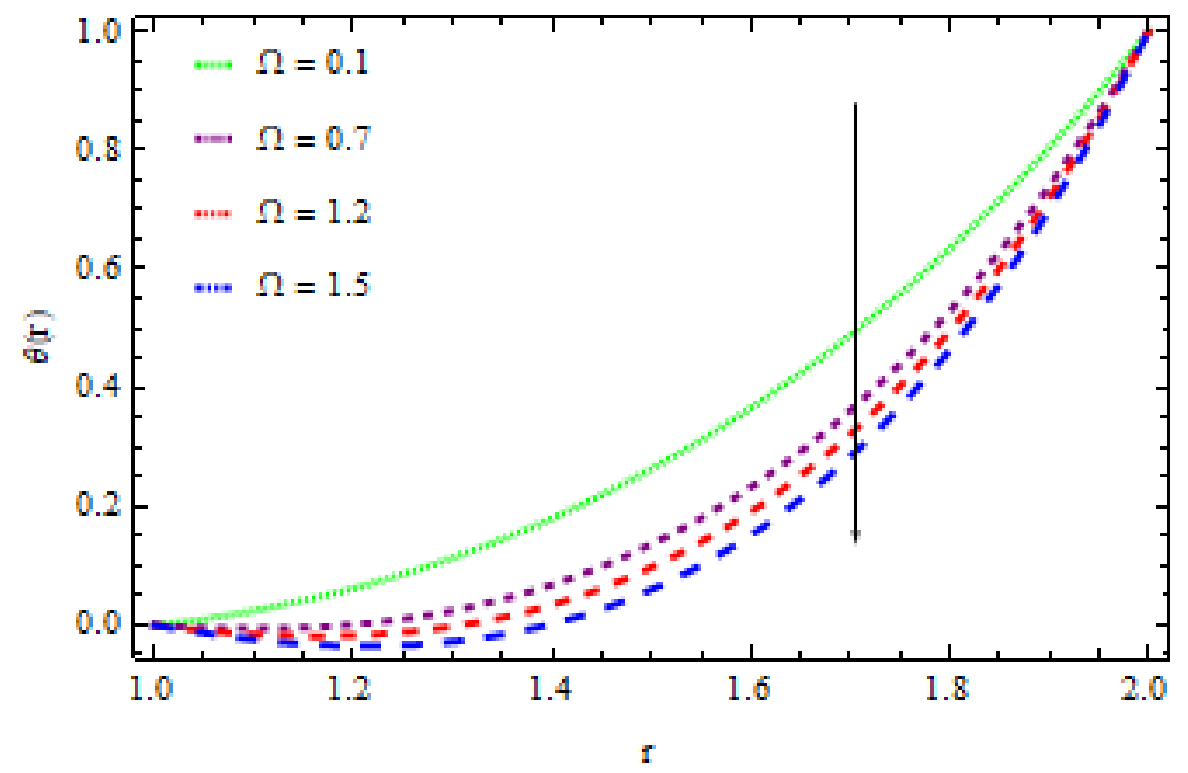

Figure 15. The influence of $\Omega$ on temperature distribution for Vogel's model (VM) when $B^{\prime}=1.2, Q=$ $0.5, D=0.5, B_{r}=0.3, M=0.21, N=0.4, K_{p}=0.5$.

\section{Conclusions}

In this paper, we studied the effects of magnetohydrodynamic stream movement and heat conduction in wire coating development using liquid polymers in a permeable medium along with a Joule heating effect and fluctuating viscosity. The wire is layered into a pressure type die to interact with the Eyring-Powell liquid. A permeable matrix is used as a dielectric to enhance the heating/cooling process and to reduce the Joule heating effect during the flow and heat conduction process. The result is derived from the Runge-Kutta 4th-order method and sketched on velocity and temperature profiles. The results are also verified by utilizing the semi-numerical method HAM and BVPh2, and these methods also find good agreement. Some valuable points which are obtained in this study are the following: 
1. An increasing behavior of the fluid velocity is observed as the values of $\mathrm{B}_{r}, M, \mathrm{~N}, D$, and $\varepsilon$ increase respectively and show a decreasing behavior as the values of $Q, \varepsilon$ and $K_{p}$ increase, respectively.

2. The temperature distribution displays an increasing effect as the values of $M$ and $\varepsilon$ are increased, while a decreasing effect is shown with increasing values of $Q, \varepsilon$ and $B_{r}$.

Author Contributions: Conceptualization: Z.K. and I.K.; Writing-Original draft: Z.K., H.U.R., and T.A.; Methodology: W.K. and K.N.; Formal analysis: I.K. and K.N.; Review and revision: I.K., W.K., D.B., and K.S.N.; Software: Z.K., H.U.R., T.A., I.K., and W.K. All authors have read and agreed to the published version of the manuscript.

Funding: This research received no external funding.

Acknowledgments: The authors extend their appreciation to the Deanship of Scientific Research at Majmaah University for funding this work under Project No (RGP-2019-3).

Conflicts of Interest: The authors declare no conflict of interest.

\section{References}

1. Nadeem, S.; Ahmad, S.; Muhammad, N. Cattaneo-Christov flux in the flow of a viscoelastic fluid in the presence of Newtonian heating. J. Mol. Liq. 2017, 237, 180-184. [CrossRef]

2. Ijaz, S.; Iqbal, Z.; Maraj, E.N.; Nadeem, S. Investigation of $\mathrm{Cu}-\mathrm{CuO} / \mathrm{blood}$ mediated transportation in stenosed artery with unique features for theoretical outcomes of hemodynamics. J. Mol. Liq. 2018, 254, 421-432. [CrossRef]

3. Abbas, N.; Saleem, S.; Nadeem, S.; Alderremy, A.A.; Khan, A.U. On stagnation point fow of a micro polar nanofluid past a circular cylinder with velocity and thermal slip. Results Phys. 2018, 9, 1224-1232. [CrossRef]

4. Ijaz, S.; Nadeem, S. Transportation of nanoparticles investigation as a drug agent to attenuate the atherosclerotic lesion under the wall properties impact Chaos. Solitons Fractals 2018, 112, 52-65. [CrossRef]

5. Tabassum, R.; Mehmood, R.; Nadeem, S. Impact of viscosity variation and micro rotation on oblique transport of Cu-water fluid. J. Colloid Interface Sci. 2017, 501, 304-310. [CrossRef] [PubMed]

6. Nadeem, S.; Sadaf, H. Exploration of single wall carbon nanotubes for the peristaltic motion in a curved channel with variable viscosity. J. Braz. Soc. Mech. Sci. Eng. 2017, 39, 117-125. [CrossRef]

7. Shahzadi, I.; Sadaf, H.; Nadeem, S.; Saleem, A. Bio-mathematical analysis for the peristaltic flow of single wall carbon nanotubes under the impact of variable viscosity and wall properties. Comput. Methods Programs Biomed. 2017, 139, 137-147. [CrossRef]

8. Ijaz, S.; Shahzadi, I.; Nadeem, S.; Saleem, A. A clot model examination: With impulsion of nanoparticles under influence of variable viscosity and slip efects. Commun. Theor. Phys. 2017, 68, 667. [CrossRef]

9. Ellahi, R.; Rahman, S.U.; Gulzar, M.M.; Nadeem, S.; Vafai, K. A mathematical study of non-Newtonian micropolar fluid in arterial blood flow through composite stenosis. Appl. Math. Inf. Sci. 2014, 8, 1567-1573. [CrossRef]

10. Hayat, T.; Nadeem, S. Aspects of developed heat and mass flux models on 3D flow of Eyring-Powell fluid. Results Phys. 2017, 7, 3910-3917. [CrossRef]

11. Hayat, T.; Nadeem, S. Flow of 3D Eyring-Powell fluid by utilizing Cattaneo-Christov heat flux model and chemical processes over an exponentially stretching surface. Results Phys. 2018, 8, 397-403. [CrossRef]

12. Ijaz, S.; Nadeem, S. A balloon model examination with impulsion of Cu-nanoparticles as drug agent through stenosed tapered elastic artery. J. Appl. Fluid Mech. 2017, 10, 1773-1783. [CrossRef]

13. Ijaz, S.; Nadeem, S. A biomedical solicitation examination of nanoparticles as drug agents to minimize the hemodynamics of a stenotic channel. Eur. Phys. J. Plus 2017, 132, 448. [CrossRef]

14. Saleem, S.; Nadeem, S.; Sandeep, N. A mathematical analysis of time dependent flow on a rotating cone in a rheological fluid. Propuls. Power Res. 2017, 6, 233-241. [CrossRef]

15. Mitsoulis, E. Fluid flow and heat transfer in wire coating: A review. Adv. Polym. Technol. 1986, 6, 467-487. [CrossRef]

16. Bagley, E.B.; Storey, S.H. Share rates and velocities of flow of polymers in wire-covering dies. Wire Wire Prod. $1963,38,1104$. 
17. Khan, N.A.; Sultan, F.; Khan, N.A. Heat and mass transfer of thermophoretic MHD flow of Powell-Eyring fluid over a vertical stretching sheet in the presence of chemical reaction and Joule heating. Int. J. Chem. React. Eng. 2015, 13, 37-49. [CrossRef]

18. Mahanthesh, B.; Gireesha, B.J.; Gorla, R.S.R. Unsteady three dimensional MHD flow of a nano Eyring-Powell fluid past a convectively heated stretching sheet in the presence of thermal radiation, viscous dissipation and Joule heating. J. Assoc. Arab. Univ. Basic Appl. Sci. 2017, 23, 75-84. [CrossRef]

19. Khana, N.; Sultan, F. Homogeneous-heterogeneous reactions in an Eyring-Powell fluid over a stretching sheet in a porous medium. Spec. Top. Rev. Porous Media 2016, 7, 15-25. [CrossRef]

20. Hayata, T.; Aslam, N.; Rafq, M.; Alsaadi, F.E. Hall and Joule heating effects on peristaltic flow of Powell-Eyring liquid in an inclined symmetric channel. Results Phys. 2017, 7, 518-528. [CrossRef]

21. Khan, Z.; Rasheed, H.; Alkanhal, T.A.; Muradullah Khan, I.; Tlili, I. Effect of magnetic field and heat source on upper-convected-maxwell fluid in a porous channel. Open Phys. 2018, 16, 917-928. [CrossRef]

22. Rehman, F.U.; Nadeem, S.; Rehman, H.U.; Haq, R.U. Thermo physical analysis for three-dimensional MHD stagnation-point flow of nano-material influenced by an exponential stretching surface. Results Phys. 2018, 8, 316-323. [CrossRef]

23. Muhammad, N.; Nadeem, S.; Mustafa, M.T. Impact of magnetic dipole on a thermally stratified ferrofluid past a stretch-able surface. Proc. Inst. Mech. Eng. Part E J. Process Mech. Eng. 2018, 1, 1989-1996.

24. Sadaf, H.; Akbar, M.U.; Nadeem, S. Induced magnetic field analysis for the peristaltic transport of non-Newtonian nanofluid in an annulus. Math. Comput. Simul. 2018, 148, 16-36. [CrossRef]

25. Nadeem, S.; Ahmad, S.; Muhammad, N.; Mustafa, M.T. Chemically reactive species in the flow of a Maxwell fluid. Results Phys. 2017, 7, 2607-2613. [CrossRef]

26. Khan, Z.; Rasheed, H.; Muradullah Gul, T.; Jan, A. Analytical and numerical solutions of Oldroyd 8-constant fluid in double-layer optical fiber coating. J. Coat. Technol. Res. 2018. [CrossRef]

27. Rehman, A.U.; Mehmood, R.; Nadeem, S. Entropy analysis of radioactive rotating nanofluid with thermal slip. Appl. Therm. Eng. 2017, 1122, 832-840. [CrossRef]

28. Muhammad, N.; Nadeem, S. Ferrite nanoparticles Ni- $\mathrm{ZnFe}_{2} \mathrm{O}_{4}, \mathrm{Mn}-\mathrm{ZnFe} \mathrm{O}_{4}$ and $\mathrm{Fe}_{2} \mathrm{O}_{4}$ in the flow of ferromagnetic nanofluid. Eur. Phys. J. Plus 2017, 132, 312-321. [CrossRef]

29. Ijaza, S.; Nadeem, S. Consequences of blood mediated nano transportation as drug agent to attenuate the atherosclerotic lesions with permeability impacts. J. Mol. Liq. 2018, 262, 565-575. [CrossRef]

30. Khan, Z.; Tairan, N.; Mashwani, W.K.; Rasheed,H.; Shah, H.; Khan, W. MHD and slip effect on two-immiscible third grade fluid on thin film flow over a vertical moving belt. Open Phys. 2019, 17, 1-12. [CrossRef]

31. Rashid, M.; Shahzadi, I.; Nadeem, S. Corrugated walls analysis in micro channels through porous medium under electromagnetohydrodynamic (EMHD) effects. Results Phys. 2018, 9, 171-182. [CrossRef]

32. Akbar, N.S. Double-diffusive natural convective peristaltic Prandtl flow in a porous channel saturated with a nanofluid. Heat Transf. Res. 2017, 48, 283-290. [CrossRef]

33. Shahzadi, I.; Nadeem, S. Impinging of metallic nanoparticles along with the slip effects through a porous medium with MHD. J. Braz. Soc. Mech. Sci. Eng. 2017, 39, 2535-2560. [CrossRef]

34. Rehman, F.U.; Nadeem, S. Heat transfer analysis for three-dimensional stagnation-point flow of water-based nanofluid over an exponentially stretching surface. J. Heat Transf. 2018, 140, 7. [CrossRef]

35. Khan, Z.; Rasheed, H.; Tlili, I.; Khan, I.; Abbas, T. Runge-Kutta 4th-order method analysis for viscoelastic Oldroyd 8-constant fuid used as coating material for wire with temperature dependent viscosity. Sci. Rep. 2018. [CrossRef]

36. Zeeshan, K.; Khan, W.A.; Rasheed, H.; Khan Nisar, K.S. Melting flow in wire coating of a third-grade fluid over a die using Reynolds' and Vogel's models with non-linear thermal radiation and joule heating. Materials 2019, 12, 3074. [CrossRef]

37. Khan, Z.; Rasheed, H.; Alharbi, S.O.; Ilyas, K.; Tariq, A.; Dennis, L.C.C. Manufacturing of double layer optical fiber coating using phan-thien-tanner fluid as coating material. Coatings 2019, 9, 147. [CrossRef]

38. Khan, Z.; Khan, M.A.; Islam, S.; Jan, B.; Hussain, F.; Rasheed, H.; Khan, W. Analysis of magneto-hydrodynamics flow and heat transfer of a viscoelastic fluid through porous medium in wire coating analysis. Mathematics 2017, 5, 27. [CrossRef]

39. Khan, Z.; Shah, R.A.; Islam SJan, H.; Jan, B.; Rasheed, H.; Khan, A. MHD flow and heat transfer analysis in the wire coating process using elastic-viscous. Coatings 2017, 7, 15. [CrossRef] 
40. Rasheed, H.; Khan, Z.; Khan, I.; Ching, D.L.C.; Nisar, K.S. Numerical and analytical investigation of an unsteady thin film nanofluid flow over an angular surface. Processes 2019, 7, 486. [CrossRef]

41. Satya Narayana, P.V.; Tarakaramu, N.; Moliya Akshit, S.; Ghori, J.P. MHD flow and heat transfer of an Eyring -Powell fluid over a linear stretching sheet with viscous dissipation -A numerical study. Front. Heat Mass Transf. 2017, 9, 9. [CrossRef]

42. Parmar, A.; Jain, S. MHD Powell-Eyring fluid flow with non-linear radiation and variable thermal conductivity over a permeable cylinder. Int. J. Heat Technol. 2018, 36, 56-64. [CrossRef]

(C) 2020 by the authors. Licensee MDPI, Basel, Switzerland. This article is an open access article distributed under the terms and conditions of the Creative Commons Attribution (CC BY) license (http://creativecommons.org/licenses/by/4.0/). 